\title{
MECHANISM OF DISORDER GENESIS IN CELLULOSE MICROFIBRILS
}

\section{URIJ ZABASHTA, MAXIM LAZARENKO, ALEXANDER ALEKSEEV, SERGEY TKACHEV, SVETLANA VASYLYUK, VALERIJ KOVALCHUK and LEONID BULAVIN}

\author{
Kyiv National Taras Shevchenko University, Faculty of Physics, \\ 60, Volodymyrska Str., Kyiv UA 01033, Ukraine \\ ¿Corresponding author: S. Yu.Tkachev, tkachevserg@gmail.com
}

Received November 9, 2020

\begin{abstract}
We discuss the possible mechanism of genesis of disordered regions in cellulose microfibrils. In our work, we show that obstacles stopping the growth of nanofibrils and the further deformation of nanofibrils may be the reasons for disorder. In this case, the initial prismatic shape of nanofibrils becomes corrugated and regions, where cellulose chains lose their initial straight configuration, appear in it. If a pack of thus deformed nanofibrils form a microfibril, then it contains disordered regions of two types. The first one consists in boundary layers, which separate nanofibrils from each other. The second type represents amorphous regions located inside each nanofibril. In this discussion, we use the linear theory of elasticity. With its help, we estimated longitudinal dimensions of cellulose crystallites and then compared them with values of such sizes calculated based on experimental data. The experimentally obtained values turned out to be, to a certain extent, greater than the theoretically estimated ones.
\end{abstract}

Keywords: cellulose structure, structure formation, deformation, nano- and microfibrils, crystal and amorphous cellulose

\section{INTRODUCTION}

A microfibril is a textural unit of cellulose. ${ }^{1}$ There are different structural models of microfibrils described in existing studies. ${ }^{2-7}$ In this work, we discuss the model of amorphouscrystalline microfibrils with straight chains. ${ }^{5-7}$ Authors quite often use this model for interpreting experimental data. ${ }^{8-12}$ This model is usually considered as the most consistent with experimental data. ${ }^{7,8}$

The model is illustrated in Figure 1a. In it, we consider a microfibril as a bunch of nanofibrils (N) separated from each other by disordered

boundary layers (B). A nanofibril contains alternating crystal (C) and amorphous (A) regions. The chains that form nanofibrils do not have folds (Fig. 1b). The chains in A-regions are the prolongations of the chains that form neighboring $\mathrm{C}$-regions and connect them.

As the reader can see in Figure 1, a microfibril contains two types of disordered regions: Aregions and B-layers. In this work, we propose and discuss the possible physical mechanisms of the genesis of these regions. As far as we know, nobody has studied this issue yet.
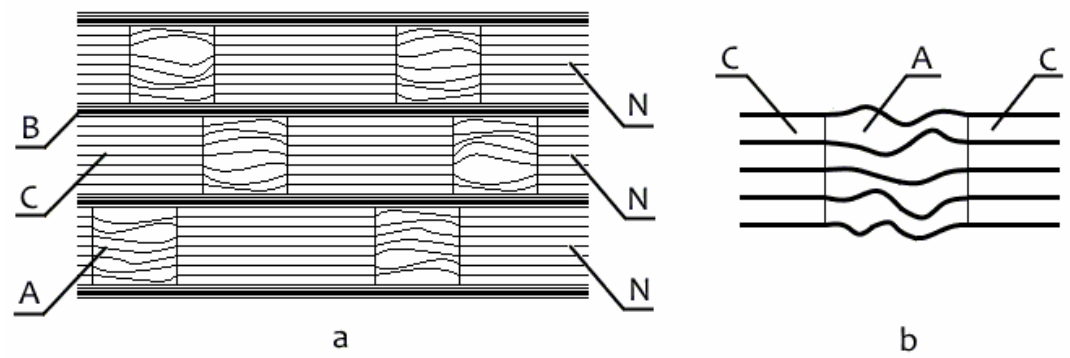

Figure 1: Scheme illustrating the structure of a microfibril: a - structural regions in a microfibril, $\mathrm{b}-$ arrangement of chains in nanofibrils 


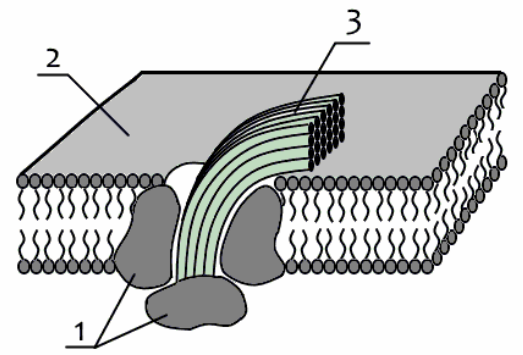

Figure 2: Formation of a nanofibril: 1 - terminal complex, 2 - plasmalemma, 3 - nanofibril

\section{Proposed models}

Instability of a nanofibril's prismatic form, occurring when a nanofibril interacts with obstacles

According to M. Brown, ${ }^{13,14}$ the formation (biosynthesis, polymerization) of cellulose molecules happens in the enzyme complex 1 (Fig. 2 ), which is located in plasmalemma 2 of a cell and has the form of a socket.

Complex 1 is usually denoted as TC (terminal complex). In this complex, both the polymerization and crystallization of the formed chain take place. As a result, a nanofibril, which grows parallel to the plasmalemma surface, is formed at the exit.

A nanofibril formed in TC has the form of a prism and is an ideal crystal. During the process of the nanofibril's growth, the front end is moving on the plasmalemma surface. When moving, this end runs into obstacles. Such an obstacle could be a nanofibril generated by another TC, roughness of the plasmalemma surface and so on. The front end, having encountered an obstacle on its path, stops. We will denote the distance between the obstacle and the TC, forming the nanofibril under consideration, as $\ell_{0}$. At the moment $t=t_{0}$ of the nanofibril's meeting the obstacle, the nanofibril has the same length, $\ell_{0}$.

If the TC this nanofibril belongs to prolongs to generate a crystalline structure, then, at moment $t$, provided there is no obstacle, the length of nanofibril $\ell$ should become equal to:

$\ell=v\left(t-t_{0}\right)$

where $v$ is the velocity of motion of the nanofibril's front end.

However, the distance $\ell_{0}$ between the TC and an obstacle is constant, so the length of a nanofibril cannot be greater than $\ell_{0}$ and, accordingly, the force $P^{\prime}$ that shrinks the nanofibril in its longitudinal axis direction, arises.
As a result, the nanofibril is deformed, shortening by $\Delta \ell=\ell-\ell_{0}$. Until the moment $t_{0}$, there is no stress in the nanofibril. Due to the deformation, stress arises in the nanofibril from this moment, and its value increases over time. When this stress is relatively small, the nanofibril retains its crystalline structure throughout its volume, as well as its prismatic form set by TC. However, compression could reach some critical $P_{\mathrm{C}}^{\prime}$ value, at which the prismatic form of the nanofibril becomes unstable.

An approximate approach to describe the structural changes occurring in a nanofibril when it interacts with obstacles

The crystalline structure of nanofibrils determines their prismatic form. Therefore, the loss of stability of the prismatic form inevitably entails structural changes, which are reduced to the appearance of disordered regions in the nanofibril.

Ideally, to describe structural changes in a nanofibril, it would be necessary to use a microscopic model. In this case, in the structure model of the system, it is necessary to set the value of the radius vector $\vec{q}_{J}$, which determines the position of each of the $N$ atoms that make up the system or, in other words, set the values of the multidimensional vector $\vec{Q} \equiv\left\{\vec{q}_{J}\right\}$, for which $J=1,2, \ldots, N$.

We denote as $\vec{Q}_{0}$ the value of the vector for a nanofibril after its collision with an obstacle. This value is the solution of the following equation: ${ }^{15}$

$\frac{\partial W(\vec{Q})}{\partial \vec{Q}}=0$

where $W(\vec{Q})$ is the potential energy of the system. 
At first glance, the method for determining the structure related to the solution of Equation (2) is exact. However, its accuracy worsens with the lack of accurate information about atomic-atomic potentials: the choice of the latter is largely arbitrary.

In connection with this circumstance, we use another computational model in this work, which we call a microscopic continuum model. Using this model, we will perform a description of the structural changes of nanofibrils in two stages. In the first stage, we introduce the continuum model of nanofibrils and use this model to determine how the shape of the nanofibril surface changes when it encounters an obstacle. In the second stage, we determine the configurations of the chains that fit into the nanofibril circuit, calculated using the continuum model.

Carrying out this program, we will consider a nanofibril with its chain structure (Fig. 3a) as a section of the elastic continuum, which has the shape of a prism (Fig. 3b). Since, as already mentioned, we will be interested in changes in the surface of a nanofibril, we simplify the model presented in Figure $3 \mathrm{~b}$ for this purpose.

As the origin, we choose a point equidistant from the side faces of the nanofibril, directing the axes $\mathrm{X}$ and $\mathrm{Y}$ horizontally and vertically perpendicular to the side faces, and the axis $\mathrm{Z}$ parallel to the direction of the chains (Fig. 3a). As can be seen from this figure, the nanofibril's cross-section is symmetrical: the axes $\mathrm{X}$ and $\mathrm{Y}$ are axes of symmetry of the second order. We assume that, during deformation, the mentioned symmetry of the cross-section is preserved. During deformation, the cross-section undergoes a similarity transformation (Fig. 3b, where the cross-section after deformation is shown with a dashed line).

We will also consider that vertical (in the direction of the axis $\mathrm{Y}$ ) and horizontal (in the direction of the axis $\mathrm{X}$ ) deformations are independent. To describe the vertical deformation, we introduce the continuum model shown in Figure $4 \mathrm{a}$. At the indicated deformation, the plane $X 0 Z$ remains motionless.

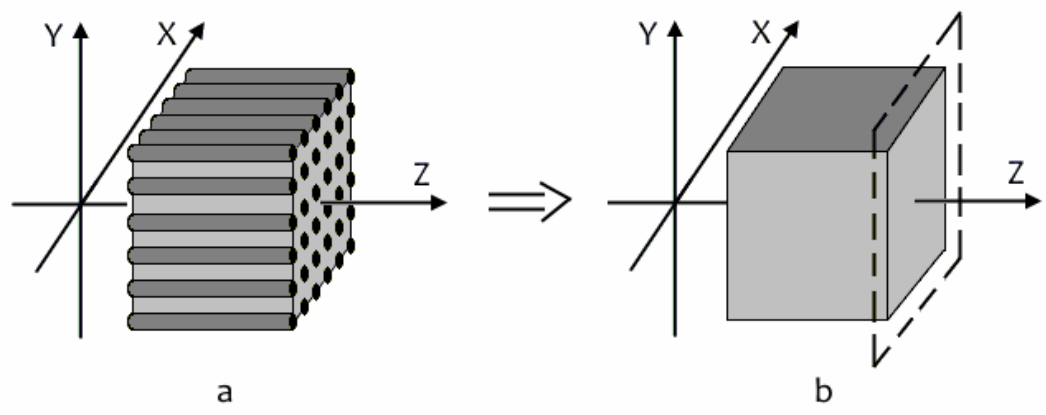

Figure 3: Replacement of the chain structure of nanofibrils (a) by an elastic continuum (b)
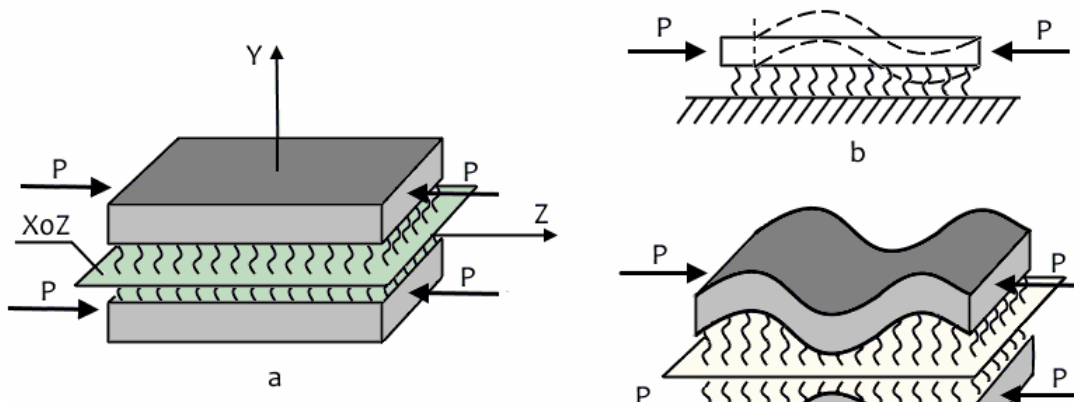

$\mathrm{b}$

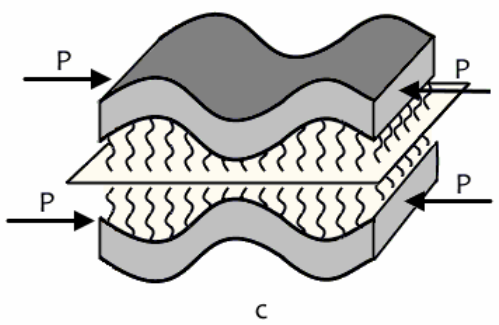

Figure 4: The continuum model (a) describing the vertical deformation (b, c) of a nanofibril 
Accordingly, the nanofibril is divided into two halves: the upper and lower, each of which could be represented as an elastic rod with a thickness $h=b / 2$ and width $b$, where $\mathrm{b}$ is the transverse size of the nanofibrils. Each rod is connected to a fixed plane by elastic bonds continuously distributed along this plane and is subjected to a compressive force $P$, which acts along the longitudinal axis of the rod. The length of the rod in the undeformed state is $\ell_{0}$.

\section{A change in the surface shape of a nanofibril occurring when it interacts with an obstacle}

With the introduction of the model shown in Figure $4 \mathrm{a}$, consideration is focused on the problem, well-known in the theory of elasticity, of stability of an elastic rod lying on an elastic base. $^{16}$

The equilibrium equation of such a rod has the following form:

$$
E J \frac{d^{4} u}{d z^{4}}+|P| \frac{d^{2} u}{d z^{2}}+K u=0
$$

where $E$ is the elastic modulus, $J=b h^{3} / 12$ is the moment of inertia of the cross-section of the rod, $K$ is the elastic coefficient of the solid base, $u$ is the deviation of the points of the middle plane of the rod from the axis $Z$.
When the compressive force $P$ reaches a critical value $P_{\mathrm{C}}$, a non-zero solution of Equation (3) appears, which has the following form:

$u \sim \sin \frac{m \pi z}{\ell_{0}}$

where $m$ is an integer. The deformation of the upper rod corresponding to this formula is shown in Figure $4 b$.

As can be seen from the formula above (Eq. 4), the rod at $P=P_{\mathrm{C}}$ is a sinusoid consisting of $m$ half-waves. The following equality is used ${ }^{16}$ to determine the number of half-waves:

$$
\frac{K \ell_{0}^{4}}{E J \pi^{4}}=m^{2}(m+1)^{2}
$$

Introducing the notation $L=\ell_{0} / m$, we rewrite equality (5) in the form:

$L=\pi\left(\frac{E J}{K}\right)^{1 / 4}\left(1+\frac{2}{m}+\frac{1}{m^{2}}\right)^{1 / 4}$

A deformation similar to the deformation of the upper rod also occurs with the lower rod, so that, in general, the continuum model (Fig. 4a) under the action of the compressive force takes the form shown in Figure 4c.

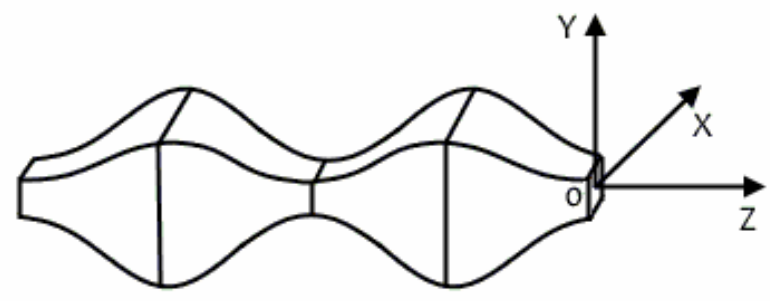

Figure 5: Shape of a nanofibril's surface after it encounters an obstacle

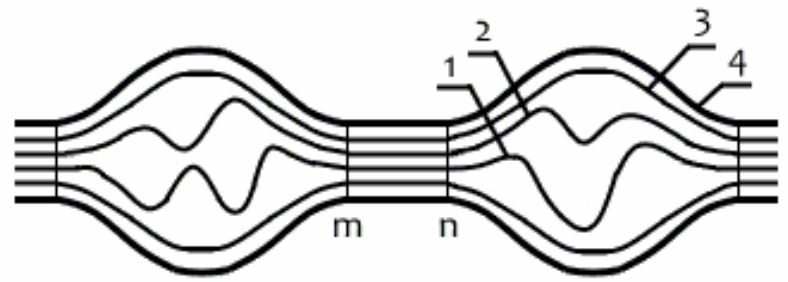

Figure 6: Configuration of the chains in the nanofibril that experienced contact with an obstacle

The model also describes horizontal deformation shown in Figure 4, with the difference that the displacements $u$ are now horizontal, i.e., directed along the axis $X$. Adding horizontal and vertical displacements, we conclude that, after stopping because of an 
obstacle, the surface of a nanofibril takes the form shown in Figure 5.

Changes in the configurations of nanofibril chains arising from its interaction with an obstacle

We use the continuum model to establish that, in the deformed state that occurs after the collision of nanofibrils with an obstacle, humps and troughs appear on its surface: the deformed surface resembles a corrugated pipe, the transverse size of which $D$ varies periodically along the axis of the pipe with a period equal to $2 L$.

We will consider the smallest value of the mentioned size equal to the thickness $b$ of the undeformed fibril, assign the size $L$ to the trough, and assume that the pipe's transverse dimension remains constant and equal $b$ in this section. Under these assumptions, the surface of the deformed fibril in its section by the plane $Y 0 Z$ will have the form depicted by the bold line 4 in Figure 6.

Assuming the mentioned surface is rigidly fixed, let us consider what configuration the chains have, located inside the space bounded by this surface.

In areas where $D=b$, the chains have nothing else to do, so they are located parallel to the pipe axis (section $\mathrm{mn}$ in Fig. 6). In other words, the initial crystalline structure of the nanofibrils is retained in this region, i.e., this region is a C-region, crystallite. As follows from the previous reasoning, its length is equal to $L$.

In areas where $D>b$, each chain experiences bulging, deviates from a line that is an extension of the axis of this chain in the adjacent crystallite. The distance of the mentioned line from the axis $Z$ is denoted by $r$. The transverse displacement of the chain from this line in the region where $D>b$ is denoted by $\Delta$. For each chain in the region $D>b$, the displacement $\Delta$ depends on $z$. The value $r$ is a parameter identifying the chain in this area. Accordingly, we represent $\Delta$ as a function of $r$ and $z$ :

$\Delta=\Delta(r, z)$

The largest displacement value for this chain is denoted by $\Delta_{\mathrm{m}}, \Delta_{\mathrm{m}}$ depends on $r$, and the function:

$\Delta_{\mathrm{m}}=\Delta_{\mathrm{m}}(r)$

monotonously decreases as $r$ decreases.
Indeed, nanofibrils, for which $r=b / 2$, have no neighbors. Therefore, for them, the value $\Delta_{\mathrm{m}}$ is the largest compared to the chains for which $r<b / 2$. Since the influence of the surface decreases with the distance from it, it is logical to assume that the value $\Delta_{\mathrm{m}}$ decreases correspondingly.

Under the accepted model, a section for which $D>b$ formed during longitudinal compression of a nanofibril from a section that contains parallel straightened chains. Therefore, the length of the segments of the chains forming the region $D>b$ is the same.

However, the values $\Delta_{\mathrm{m}}$, as already mentioned, are different for different chains. With the same length of the mentioned chain segments, this difference can be achieved if the chains in the region $D>b$ have different configurations.

The chains with a low value of $r$, for which the bulging possibilities are limited, compensate for the excess length formed during the longitudinal compression of the nanofibril by the formation of several bends. For such chains, the function $\Delta(r, z)$ has several extrema (chains 1,2 in Fig. 6), and the greatest displacement satisfies the inequality:

$\Delta_{\mathrm{m}}<b / 2$

We will denote these chains by $A_{1}$.

The chains with values of $r$ close to $b / 2$ (chains 3, 4 in Fig. 6), being able to bulge out relatively freely, have only one extremum and are characterized by inequality:

$\Delta_{\mathrm{m}}>b / 2$

We will denote such chains by $B_{1}$.

\section{Structural model of microfibrils}

A microfibril is known to be formed as a result of the aggregation of nanofibrils. As can be seen from Figure 6, the surface of the nanofibrils is corrugated. Therefore, to form tight packing, nanofibrils are located in microfibrils, so that one nanofibril's protrusions enter the troughs of another (Fig. 7).

In each nanofibril, we draw a surface $S$ that coincides with the boundary surfaces of the crystallites of a nanofibril. In areas where the surface $S$ coincides with the crystallite boundary, this surface is a plane located at a distance $b / 2$ from the axis of a chosen nanofibril. Due to protrusions on the surface of nanofibrils, a layer, 
filled with $B_{1}$-chains, arises between the surfaces $S$ of adjacent nanofibrils. This layer is a boundary layer $B$, separating nanofibrils from each other in the model shown in Figure 1 (in Fig. 7 , the $B$-layers are shaded). The disordered regions formed by $A_{1}$-chains that remain inside the surface of each nanofibril (Fig. 7) are nothing more than amorphous regions ( $A$-regions) in the model depicted in Figure 1.

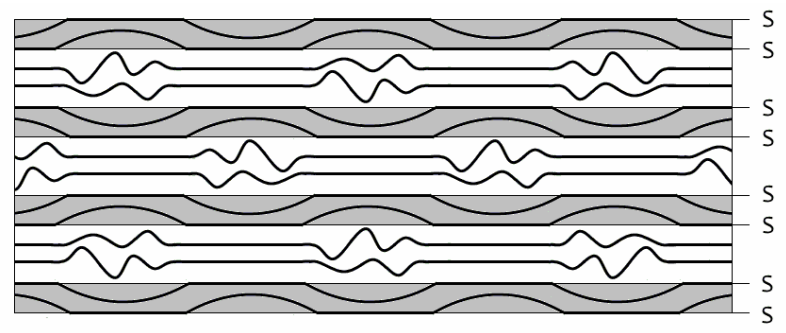

Figure 7: Aggregation of nanofibrils, leading to the formation of microfibrils

\section{RESULTS AND DISCUSSION}

The opportunity to check whether the proposed mechanism of disorder is consistent with experiment is given to us by formula (6). Using this formula, we calculate the crystallite length $L_{\text {teor }}=L$ and compare the obtained values with the value determined from the experiment $\left(L_{\text {exp }}\right)$.

First, we estimate the values of the quantities included in formula (6), based on literature data. In its physical sense, the coefficient value $K$ is determined by intermolecular interaction. For cellulose, this interaction is realized through hydrogen bonds. Therefore, it is evident that the mentioned coefficient should be a function of $\mathcal{E}$, the hydrogen bond energy. Considering the dimension of this coefficient $\left[\mathrm{N} / \mathrm{m}^{2}\right]$, it follows from the previous sentence that this coefficient represents the energy density of intermolecular interaction:

$K \approx \mathcal{E} / v$

where $v$ is the volume per one hydrogen bond. We can estimate this volume using the ratio:

$v \approx c d^{2}$

where $c$ is the distance between the hydrogen bonds connecting adjacent chains, $d$ is the distance between the chains.

Each glucose residue forms one hydrogen bond with an adjacent chain. ${ }^{8,17}$ The cellulose crystal cell contains two such residues, which form hydrogen bonds with different neighboring chains. Therefore, for hydrogen bonds connecting this chain with one of the neighboring chains, the distance $c$ is equal to the cell's size along the axis of the chains $c=10.4 \AA$.

We obtain an estimate from the unit cell structure data: $d \approx 8 \AA \AA^{8,17,18}$ The value of the hydrogen bond energy $\varepsilon=4.1 \cdot 10^{-20} \mathrm{~J}$ is given in an earlier study. ${ }^{19}$ Substituting the given numerical values in formulas (12) and (11), we obtain the estimate $K \approx 6.2 \cdot 10^{7} \mathrm{~Pa}$. The value of the elastic modulus $\mathrm{Pa}$ is given by R.J. Moon et $a l^{20}$

For the moment of inertia of the cross-section of the rod, we have equality $J=b^{4} / 96$. Assuming that the number of half-waves $m$ is not more than 2 , we can estimate the multiplier value $\left(1+2 / m+1 / m^{2}\right)^{1 / 4} \approx 1,1$. Substituting the numerical data into formula (6) and taking into account that, in the model under consideration, the quantity $b$ is identified with the transverse crystallite size $L_{\perp}$, we obtain the estimate:

$L_{\text {teor }}=L \approx 9 L_{\perp}$

Table 1 shows the experimental ${ }^{8}\left(L_{\perp}, L_{\text {exp }}\right)$ and calculated ( $L_{\text {teor }}$ ) by Equation (13) values of the length (longitudinal size) of a crystallite. The reader can see from the table that the experimental values $L_{\text {exp }}$ of the longitudinal crystallite size exceed, to a certain extent, the calculated values $L_{\text {teor }}$. 
Table 1

Transverse $\left(L_{\perp}\right)$ and longitudinal ( $L_{\text {exp }}$ ) sizes of cellulose crystallites, ${ }^{8}$ estimation of longitudinal ( $L_{\text {teor }}$ ) sizes of cellulose crystallites

\begin{tabular}{lccc}
\hline Material & $L_{\perp}, \mathrm{nm}$ & $L_{\text {exp }}, \mathrm{nm}$ & $L_{\text {teor }}, \mathrm{nm}$ \\
\hline Wheat straw cellulose & 3.4 & 53 & 30 \\
Bagasse cellulose & 3.5 & 56 & 31 \\
Birch cellulose & 3.6 & 68 & 32 \\
Spruce cellulose & 3.7 & 71 & 33 \\
Pine cellulose & 3.8 & 73 & 34 \\
Flax cellulose & 5.2 & 85 & 47 \\
Cotton lint cellulose & 6.0 & 92 & 54 \\
\hline
\end{tabular}

Nevertheless, this discrepancy should not be considered an argument that contradicts the proposed model for the genesis of disorder. The fact is that this model greatly simplifies the real situation. Not to mention that the calculations were performed in the continuum approximation - when an elastic continuum replaces the chain structure of cellulose. It is also significant that this approximation is linear: the article uses the linear theory of elasticity. In this theory, the deformed state of the rod caused by a critical compressive force is a state of unstable equilibrium, which corresponds to the value $L_{\text {teor }}$ from Equation (6). However, even insignificant deformation is sufficient for the rod to transfer from this state to a new deformed state - a state of stable equilibrium, for which the crystallite length should be somewhat larger than the value calculated by formula (6). It is such a stable state that it is realized in reality and, naturally, that $L_{\text {exp }}$ turns out to be greater than $L_{\text {teor }}$, which is what the experimental data from Table 1 demonstrate. To describe the latter, it is necessary to use a more accurate nonlinear approximation of the proposed model, based on the nonlinear theory of elasticity. ${ }^{21,22}$ Accordingly, the values $L_{\text {teor }}$ calculated in this paper should be considered as a lower estimate of the values that the cellulose crystallite length can take.

\section{CONCLUSION}

The study described in this paper allows, as we think, to state that the disorder in cellulose microfibrils arises as follows.

The biosynthesis of cellulose chains occurs in terminal complexes located in the plasmalemma of cells. In the same complexes, the chains crystallize, forming nanofibrils. A nanofibril is an ideal crystal, which has a prismatic shape.

During the operation of the terminal complex, the nanofibril grows, moving along the plasmalemma surface. Then, the nanofibril front end meets an obstacle and stops. As the terminal complex continues its work, a force arises, compressing the nanofibril in the longitudinal direction. When this force exceeds a specific critical value, the nanofibril's prismatic shape becomes unstable, and the surface of the nanofibril becomes corrugated, representing a periodic sequence of humps and troughs.

In the area corresponding to the trough, the initial crystalline structure of nanofibrils is preserved. In the area corresponding to the hump, the crystalline structure is lost, and the chains acquire a curved configuration. We distinguish two types of the curved chains: chains of the first type have a configuration that is a curve with one extremum, chains of the second type are characterized by a configuration that is a curve with several extrema.

A microfibril is formed as a result of the packing of nanofibrils. It occurs in a way that the humps of each nanofibril's surface enter the troughs of the surface of adjacent nanofibrils. As a result of this packing, two types of disordered regions arise.

The first type is represented by the boundary layers that separate nanofibrils from each other. These areas are formed by chains with a curved configuration of the first type. The disordered regions of the second type are amorphous regions located inside nanofibrils, alternating with crystalline regions. These amorphous regions are formed by chains of the second type. The latter connect adjacent crystalline regions. 
The proposed mechanism for the genesis of disorder corresponds to the model of amorphouscrystalline microfibril with straightened chains.

\section{REFERENCES}

J. H. Kim, B. S. Shim, H. S. Kim, Y. J. Lee, S. K. Min et al., Int. J. Precis. Eng. Manuf.-Green Technol., 2, 197 (2015), https://doi.org/10.1007/s40684-0150024-9

2 I. Hearle, J. Appl. Polym. Sci., 7, 1175 (1963), https://doi.org/10.1002/app.1963.070070401

3 C. Verlhac, J. Dedier and H. Chanzy, J. Polym. Sci., Part A: Polym. Chem., 28, 1171 (1990), https://doi.org/10.1002/pola.1990.080280517

4 R. Manley, Trend, 5, 4 (1965)

5 S. Azizi, M. A. S. Alloin and A. Dufresne, Biomacromolecules, 6, $612 \quad$ (2005), https://doi.org/10.1021/bm0493685

6 Y. Nishiyama, U. J. Kim, D. Y. Kim, K. S. Katsumata and R. P. May, Biomacromolecules, 4, 1013 (2003), https://doi.org/10.1021/bm025772x

7 M. Mariano, N. El. Kissi and A. Dufresne, $J$. Polym. Sci., Part B: Polym. Phys., 52, 791 (2014), https://doi.org/10.1002/polb.23490

8 M. Y. Ioelovich, Polym. Sci. Series A, 58, 925 (2016), https://doi.org/10.1134/S0965545X16060109

9 K. M. Kovalov, O. M. Alekseev, M. M. Lazarenko, Y. F. Zabashta, Y. E. Grabovskii et al., Nanoscale Res. Lett., 12, 468 (2017), https://doi.org/10.1186/s11671017-2231-5

10 A. F. Turbak, F. W. Snyder and K. R. Sandberg, J. Appl. Polym. Sci.: Appl. Polym. Symp., 37 (1983)

1 M. Nedielko, S. Hamamda, O. Alekseev, V. Chornii and M. Dashevskii, Nanoscale Res. Lett., 12, 98 (2017), https://doi.org/10.1186/s11671-017-1862-x

12 M. Lazarenko, A. Alekseev, Yu. Zabashta, S. Tkachev, V. Kovalchuk et al., Cellulose Chem. Technol., $\quad 54, \quad 199 \quad$ (2020), https://doi.org/10.35812/CelluloseChemTechnol.2020. $\underline{54.22}$
13 M. Brown, J. Macromol. Sci., Part A: Pure Appl. Chem., 33, $1345 \quad$ (1996), https://doi.org/10.1080/10601329608014912

14 M. Brown, J. Polym. Sci., Part A: Polym. Chem., 42, 484 (2004), https://doi.org/10.1002/pola.10877

15 L. D. Landau and E. M. Lifshitz, "Mechanics", Elsevier, Butterworth-Heinemann, 1982

16 L. D. Landau, L. P. Pitaevskii, A. M. Kosevich and E. M. Lifshitz, "Theory of Elasticity", Elsevier, Butterworth-Heinemann, 2012

17 A. D. French, Cellulose, 21, 885 (2014), https://doi.org/10.1007/s10570-013-0030-4

18 Y. Habibi, L. A. Lucia and O. J. Rojas, Chem. Rev., 110, 3479 (2010), https://doi.org/10.1021/cr900339w

19 O. M. Alekseev, K. M. Kovalov, M. M. Lazarenko, M. V. Lazarenko, Y. E. Grabovskii et al., Cellulose Chem. Technol., 53, $15 \quad$ (2019), https://doi.org/10.35812/CelluloseChemTechnol.2019. 53.02

20 R. J. Moon, A. Martini, J. Nairn, J. Simonsen and J. Youngblood, Chem. Soc. Rev., 40, 3941 (2011), https://doi.org/10.1039/c0cs00108b

21 A. I. Lurie, "Non-Linear Theory of Elasticity", Elsevier, North Holland, 1990

22 L. A. Taber, "Nonlinear Theory of Elasticity: Applications in Biomechanics", World Scientific, 2004 